\title{
Obsessive compulsive phenomenology in a sample of Egyptian adolescent population
}

\author{
Ahmed Rady, MD*,** \\ Hoda Salama, MD*,** \\ Mervat Wagdy, MD*** \\ Ahmed Ketat, MD** \\ * Department of Psychiatry, Alexandria \\ University \\ ** Alexandria University Hospitals, \\ Alexandria \\ ${ }^{\star * *}$ High Institute of Public Health, \\ Alexandria \\ EGYPT
}

\begin{abstract}
Background and Objectives: Obsessive symptoms among children and adolescent age groups are increasing, an observation made by mental health professionals working with this age group. Our epidemiological study targeted secondary school students to estimate the prevalence of obsessive symptoms, obsessive compulsive disorder and their different obsessive compulsive contents.

Methods: The study is cross sectional carried on 1299 secondary school students, the sample size was chosen based on an estimated Obsessive Compulsive Disorder (OCD) prevalence of $2 \%$ in literature. Equal samples were recruited from the 3 educative zones in Alexandria Governorate. Obsessive compulsive symptoms were assessed by the Arabic version of Lyeton obsessive inventory child version LOI-CV. Students scoring above 35 were subjected to the Mini International Neuropsychiatric Interview for children MINIKID Arabic. OCD patient students detected by MINI-KID were assessed by psychiatric interview to confirm fulfilling criteria of OCD according to DSM IV -TR criteria. Different obsessive compulsive symptoms were assessed by a standardized questionnaire.

Results: Among the studied sample ( $\mathrm{n}=1299), 201$ students scored $>35$ on LOI-CV i.e. $15.5 \%$ of the total sample have OCS. The prevalence of OCD among studied sample was $2.2 \%$ as 29 students from the OCS students were fulfilling diagnostic criteria for OCD according to DSM-IV TR. Common obsessive symptoms were of excessive conscience $65.5 \%$, blasphemous $55.2 \%$, repeated words $51.7 \%$ and sexual obsessions $48.2 \%$.

Conclusions: The prevalence of obsessive compulsive symptoms is high among adolescent age group. Cultural impact should be considered to better understand obsessive phenomenology, raising the importance of OCD study from a transcultural perspective.
\end{abstract}




\section{Introduction}

The World Health Organization rates OCD as one of the top 20 most disabling diseases ${ }^{1,2}$. Though it may persist if left untreated, yet effective and evidence based psychological as well as drug treatments are available ${ }^{3-5}$. Early epidemiological studies report prevalence rates of $0.8 \%$ in adults and $0.25 \%$ in 5-15 year old children. In the last decade, the prevalence of OCD symptoms in the general population has been found to be remarkably high ${ }^{6-8}$. Until 1984 the most quoted figure was $0.05 \%$.

However, since 1984, 3 studies carried out in North America found prevalence of OCD in the general population to be greater than $2 \%$ which is 40 times higher than the earlier estimation from 1950. A multicentre study carried out in to assess OCD prevalence to be approximately $2 \%$ in the USA, Canada, Latin America \& Puerto Rico, the findings where the same in Europe and new Zealand, while in Asia it was found to be $1.9 \%{ }^{9-12}$.

OCD is a global problem, as the estimated total number of population who suffered from the disorder appears to be at least 50 million worldwide ${ }^{13}$. OCD is ranked as $2^{\text {nd }}$ most prevalent psychiatric disorder, although that it is still underestimated worldwide, it may be the ego dystonic nature of the disorder that enforce the sufferer to disguise or be ashamed from their symptoms, and they will not reveal their obsessive and or compulsive symptoms till they are asked directly! Young people with the disorder perceive their symptoms as embarrassing and do not disclose them unless specifically asked.

Therefore, OCD in this age group often remains unrecognized and untreated. The associated distress and developmental handicap are avoidable as effective treatments are available. There is evidence that early detection and intervention improve outcome ${ }^{13,14}$.
The aim of this work is to epidemiologically assess the prevalence of obsessive compulsive symptoms, obsessive compulsive disorder among secondary school students and to study different presenting obsessive and compulsive symptoms.

\section{Subjects and methods}

Cross sectional study design was adopted. The study sample was calculated using the computer package: Epi-Info, based on an OCD prevalence estimate of $2 \%$ in literatures. The determined minimum sample size required was 1299 students. Type I statistical error alpha was set at 0.05 . Out of the 7 educational zones in Alexandria three zones with the highest density of secondary school students were included in the study namely; East, Middle and West educational zones. The predetermined sample was equally allocated on the selected educational zones. Each selected zone was represented by one public school for boys and another one for girls and a mixed sex private school. One class represented each grade level in the selected schools and the equally allocated sample on each class was selected at random. All students willing to participate in the study were included. The study was conducted on the academic year from January 2009 to December 2009.

A predesigned structured self-administered questionnaire was used to collect sociodemographic data including age, sex and grade level and socioeconomic data including family income, individual allowance, educational level of parents, occupation of parents, number of rooms and electrical appliances in home for keeping in registry.

Psychological assessment using the Arabic Version of Leyton Obsessive Inventory-Child Version (LOI-CV). It is a self-administered 
scale that was designed to measure obsessive compulsive symptoms and traits in children and adolescents. It is a modification of the original Leyton Obsessional Inventory, which was modified by Berg et al. LOI-CV consists of inventory 20 questions covering: thoughts, dirt \& contamination, cleanliness \& tidiness, order \& routine, over conscience, checking, school work, repetition, and indecision. Scoring of items ranges between 0-3 as follows: $0=$ the child do not have the symptoms. $1=$ the symptom is present with minimal interference in daily activities. $2=$ the symptom is present with moderate interference. $3=$ the symptom is present with severe interference. The total score ranges from 0 to 60 . According to authors, high scorers were identified at cutoff score $34 / 35$. The high scorers are students with obsessive compulsive symptoms OCSs $^{15-17}$.

Psychiatric assessment using Mini International Neuropsychiatric Interview for Children (MINI-KID) was used to identify psychiatric disorders among students with OCSs. This scale was originally designed by Sheehan et al. following the same structure and format of the adult version. The MINI-KID follows the DSM-IV and ICD-10 criteria for the diagnosis of psychiatric disorders and screens for 17 Axis I disorders (mood disorders, anxiety disorders including OCD, attention deficit disorder, conduct, alcohol/substance abuse or dependence, eating disorders and psychotic disorders). The MINI-KID is a reliable and valid measure of child and adolescent psychopathology that can be administered in a short time (5-15 minutes). It use the branching logic model to reduce the number of questions asked to only those necessary to determine the presence or absence of each diagnosis It's sensitivity and specificity is high (0.61-0.80) to very high $(0.81+)$ for all diagnosis. The test-retest reliability of the
MINI-KID is uniformly high to very high for all psychiatric disorders ${ }^{18}$.

All students with LOI-CV score $\geq 35$ where subjected to the Arabic Version of MINI$\mathrm{KID}^{19}$. All OCD cases recruited by MINI-KID were subjected to psychiatric assessment to verify OCD diagnosis using DSM-IV-TR criteria (American Psychiatric Association: Diagnostic and Statistical Manual of Mental Disorders revised text).

Different obsessive and compulsory behaviors were assessed by a standardized selfreporting questionnaire.

\section{Statistical analysis}

After the data were collected, they were coded then entered into the computer. The Statistical Package for Social Sciences (SPSSversion 11.5) as well as the Epidemiological Information Package (Epi Info 2002) were utilized for data analysis and tabulation of results. Appropriate descriptive statistics, arithmetic mean, median, and standard deviation were done. Statistical error type I set at a level $<0.05$.

\section{Results}

Among the studied sample $(\mathrm{n}=1299), 201$ students scored $>35$ on LOI-CV i.e. $15.5 \%$ of the total sample have obsessive compulsive symptoms OCS, revealing a prevalence of 0.155 with a standard error estimated at 0.00277 and $\mathrm{IC}_{95 \%}=[0.14957-0.16043]$. The prevalence of OCD among studied sample was $2.2 \%$ as 29 students from the OCS students were fulfilling diagnostic criteria for OCD according to DSM-IV TR with a prevalence of 0.022 , standard error of 0.00013 and $\mathrm{IC}_{95 \%}[0.02177-0.02222]$ (Figure 1). 


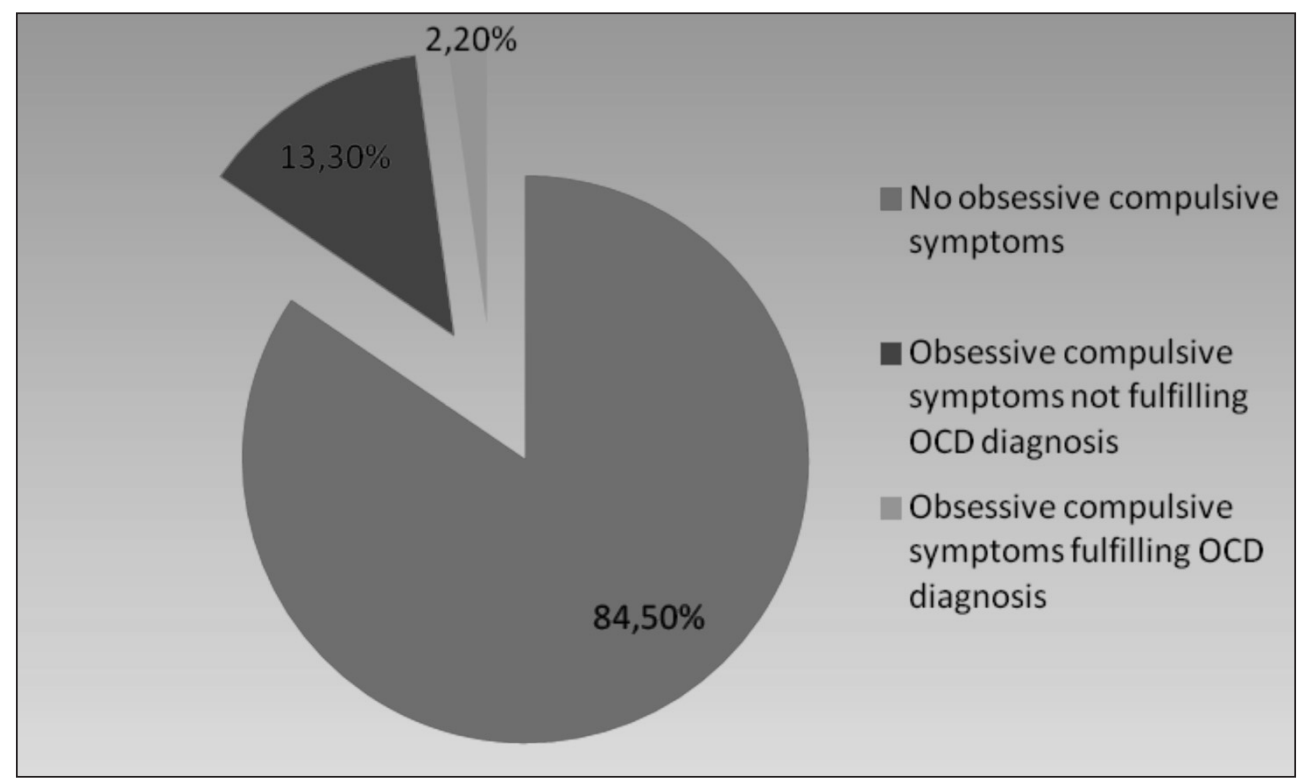

Figure 1. Prevalence of obsessive compulsive symptoms (OCS) and obsessive compulsive disorder (OCD) among adolescents in the studied sample.

Table 1

Age and gender distribution among adolescents in the whole sample, with OCS and those showing OCD

$$
\begin{gathered}
\text { Total sample } \\
(\mathrm{n}=1299)
\end{gathered}
$$

Adolescents with OCS

$$
(\mathrm{n}=201)
$$

Adolescents OCD

$$
(\mathrm{n}=29)
$$

\begin{tabular}{lccc}
\hline Age (yrs) & $16.1 \pm 1$ & $15.87 \pm 1.04$ & $15.59 \pm 1.02$ \\
\hline Gender & & & \\
Male & $49.3 \%$ & $51.7 \%$ & $44.8 \%$ \\
Female & $50.7 \%$ & $48.3 \%$ & $55.2 \%$ \\
\hline
\end{tabular}

Both groups with OCS and OCD were balanced in terms of age and gender distribution (Table 1).

Common obsessive symptoms found among OCD students were excessive conscience, blasephamous, repeated words, sexual, symmetry and contamination in $65.5 \%, 55.2 \%$, $51.7 \%, 48.2 \%, 24.1 \%$ and $24.1 \%$ respectively (Table 2). On the other hand, common compulsive symptoms found among OCD students were repetition, checking, washing, ordering and hoarding in $82.8 \%, 69 \%$, $55.2 \%, 44.8$ and $38 \%$ respectively (Table 3 ).

Distribution of different obsessive contents with gender didn't reveal significant difference $\left(X^{2}=9.04, d l=5, p>0.05\right)$. Again, gender didn't affect compulsive contents $\left(\mathrm{X}^{2}\right.$ $=8.87, \mathrm{dl}=4, \mathrm{p}>0.05)$. 
Table 2

Common obsessive symptoms among OCD students $(\mathrm{n}=29)$

\begin{tabular}{lcc} 
Obsessive symptom & Number of students & Percentage \\
\hline Repeated words & 15 & $51.7 \%$ \\
Contamination & 7 & $24.1 \%$ \\
Symmetry & 7 & $24.1 \%$ \\
Excessive conscience & 19 & $65.5 \%$ \\
Sexual & 14 & $48.2 \%$ \\
Blasphemous & 16 & $55.2 \%$ \\
\hline
\end{tabular}

Table 3

Common compulsive symptoms among OCD students $(\mathrm{n}=29)$

\begin{tabular}{lcc} 
Compulsive symptom & Number of students & Percentage \\
\hline Checking & 20 & $69 \%$ \\
Washing & 16 & $55.2 \%$ \\
Ordering & 13 & $44.8 \%$ \\
Repetition & 24 & $82.8 \%$ \\
Hoarding & 11 & $38 \%$ \\
\hline
\end{tabular}

\section{Discussion}

In this study the estimated overall prevalence of OCD among secondary schools' students was $2.2 \%$ while the prevalence for obsessive compulsive symptoms OCS was $13.3 \%$. These results fall in the range of estimates of $0.6 \%$ to $26.3 \%$ for OCD and $14.2 \%$ to $38.7 \%$ for OCS obtained from prior studies conducted among similar target population (secondary school students) ${ }^{20,21}$. A study on the prevalence of OCS in Egyptian students in Cairo (secondary schools and university students) using General Health Questionnaire, Arabic Obsessive scale, revealed that the prevalence of OCS is $38.7 \%$ in secondary schools ${ }^{20}$. On the other hand, prevalence of OCD among the secondary school students was $26.3 \%$ (51.3\% male and $48.7 \%$ females). These results are higher than our re- sults. Such difference may be attributed to difference in study population, the current study was conducted on urbain area rather than semiurbain area $^{20}$. Another two stage epidemiological study which was conducted to investigate the point prevalence rates of OCS and OCD among secondary schools in rural areas of Shebin El-Kanater, El-Kalubia governorate in Egypt using Arabic form of Symptoms Checklist "SCL-90" to screen for OCS, in the second stage, a semi structured clinical interview based on DSM-IV diagnostic criteria was performed on the students whom considered positive in the first stage. This study was conducted among 1000 students of both genders of almost equal distribution, it was found that $9.1 \%$ were fulfilling diagnostic criteria for OCD (4.4\% for male and $4.7 \%$ for females) and $18.4 \%$ were positive for OCS. The prevalence of OCD in this study was lower, this may be due to the use of different 
scale and to the fact that the sample used in the present study was distributed across different socioeconomic levels ${ }^{21}$.

In a study conducted to determine the prevalence of OCS among Egyptian students among students came from the El Abasseya educational area in Cairo. The tools used in this study included the General Health Questionnaire for screening of psychiatric morbidity and the Arabic Obsessive Scale for obsessive traits. The Y-BOCS was used to determine the profile of OCS and the ICD-10 was used for diagnosis of OCD. Prevalence rate for OCS was $43.1 \%$. Obsessive-compulsive symptoms were more prevalent among younger students, females and first-born participants. Aggressive, contamination and religious obsessions and cleaning compulsions were most common. Among participants $19 \%$ of students with OCS fulfilled ICD-10 criteria for $\mathrm{OCD}^{22}$.

As regard studies conducted among similar cultures, a Saudi study of prevalence of psychological symptoms in Saudi secondary school girls, using cross-sectional study design in 10 secondary schools for girls using the Arabic version of the symptom-revised checklist 90 (SCL 90-R) for 545 female students, OCS was found to have prevalence rate of $12.3 \%$. These results are in agreement with the result of current work despite that we conduct study among both sex and used different tool ${ }^{23}$.

Our results are in agreement with results reported by Marwa et al. where excessive conscience was reported as the most prevalent symptom $(64.7 \%)$ and the least prevalent was contamination obsessions $(33.1 \%)$, the commonest compulsions were ordering $(55.8 \%)$, checking $(35.6 \%)$ and repetition $(13.5 \%)^{24}$. The most common obsessive symptoms were excessive conscience, orderliness, contamination and repeated thoughts were also re- ported by two other studies on Egyptian adolescent population ${ }^{25,26}$.

Results were also in agreement with a study carried out on adult rather than adolescent population, showing contamination $(23.5 \%)$, religious $(33 \%)$ and pathological doubt (16.7\%) are among common obsessive symptoms while the commonest compulsion was washing (20\%). These results reflect that the clinical picture of OCD doesn't differ significantly between adults and adolescents of the same culture ${ }^{27}$.

In studies carried out on adolescents with OCD in USA, Japan and Turkey, the commonest obsession was fear of dirt (40\%) and hand washing was the most common compulsion $(85 \%)^{28-30}$. While in India the fear of harm was the commonest reported obsessions, highlighting transcultural difference ${ }^{31}$.

The similarities of the content of obsessions between Muslims and Jews compared to Hindus and Christians signify the role played by cultural and religious factors in the presentation of OCD. The obsessional contents of samples from Egypt and Jerusalem were similar, dealing mainly with matters of religion, cleanliness and $\operatorname{dirt}^{32}$. Common themes between Indian and British samples were mostly related to orderliness and aggressive issues. From the above cross cultural studies, it's obvious that cultural and religious upbringing play a crucial role in phenomenology of OCD. The religious content and themes as presenting symptoms of OCD patients from Middle East can be attributed to the value of religion as important determinant in everyday activities and rituals among Muslim countries. Though obsessions may be colored with religious tinge as repeating "wodou" cleansing before prayers, the ritualistic cleansing procedures can also be the source of obsessions and compulsions about religious purity 
or fear of hell as consequence of bad works also hide the deepest seeking for purity.

Other evidence of religious connotation inherent in OCD in Muslim culture lies in the term weswas used in reference to devil and the same term is used to describe obsessions in Arabic dialect. It's also characteristic of conservative societies like Egypt to expect sexual obsessions to be among the most frequent in female patients. Female gender is surrounded by so many religious and sexual taboos that it becomes a rich pool for worries, ruminations and cleansing compulsions ${ }^{26}$. Christians represent approximately $10 \%$ of Egyptian population, presenting symptoms were similar in terms of obsessions where religious and sexual thoughts were predominant, however, there was marked difference in rituals, more common among Muslims. This emphasizes the role of ritualistic Islamic upbringing as compared to Christian upbringing in oriental communities ${ }^{33}$.

\section{Conclusion}

The prevalence of obsessive compulsive symptoms is high among adolescent age group. Public awareness and screening in high school population may be of help for early detection and management. Religious and upbringing habits together with transcultural differences may have impact on presenting symptoms for obsessions and compulsions. Studying obsessions and compulsive behaviors from a transcultural perspective may help in better understanding of etiology, phenomenology and psychopathology. Further researches with higher population to assess the influence of age and gender on different obsessive and compulsive contents are recommended for future studies.

\section{References}

1. Flament MF, Whitaker A, Rapoport JL, Davies M, Berg $\mathrm{CZ}$, Kalikow K, et al. Obsessive compulsive disorder in adolescence: an epidemiological study. J Am Acad Child Adolesc Psychiatry 1988; 27: 412-419.

2. Zohar AH. The epidemiology of obsessive compulsive disorder in children and adolescents. Child Adolesc Psychiatr Clin N Am 1999; 8: 445-460.

3. Thomsen PH. From Thoughts to Obsessions: obsessive -compulsive disorder in children and adolescents. London: Jessica Kingsley; 1999.

4. Skoog G, Skoog I. A 40-year follow up of patients with obsessive compulsive disorder. Arch Gen Psychiatry 1999; 56: 121-127.

5. Wittchen HU, Jacobi F. Size and burden of mental disorder in Europe: a critical review and appraisal of 27 studies. Eur Neuropsychopharmacol 2005; 15: 357-376.

6. Heyman I, Fombonne E, Simmons H, Ford T, Meltzer H, Goodman R. Prevalence of obsessive-compulsive disorder in the British nationwide survey of child mental health. Br J Psychiatry 2001; 179: 324-329.

7. Karno M, Golding JM, Sorenson SB, Burnam MA. The epidemiology of obsessive-compulsive disorder in five US communities. Arch Gen Psychiatry 1988; 45: 1094-1099.

8. Regier DA, Boyd JH, Burke JD Jr, Rae DS, Myers JK, Kramer M, et al. One-month prevalence of mental disorders in the United States: based on five Epidemiologic Catchment Area sites. Arch Gen Psychiatry 1988; 45: 977-986.

9. Kessler R, Chiu W, Demler O, Walters E. Prevalence, severity, and comorbidity of 12-month DSM-IV disorders in the national Comorbidity survey replication. Arch Gen Psychiatry 2005; 62: 617-627.

10. Bijl R, Ravelli A, van Zessen G. Prevalence of psychiatric disorder in the general population: results of The Netherlands Mental Health Survey and Incidence Study (NEMESIS). Soc Psychiatry Psychiatr Epidemiol 1998; 33 : 587-595.

11. Wittchen HU, Essau CA, von Zerssen D, Krieg JC, Zaudig M. Lifetime and six-month prevalence of mental disorders in the Munich Follow-up study. Eur Arch Clin Neuroscience 199; 241(4): 247-258.

12. Lee C. Psychiatric epidemiology in Korea: Gender and age Differences in Seoul. J Ner Ment Dis 1990; 178: 242-246.

13. Stefanson J. Lifetime prevalence of specific mental disorders among people born in Iceland in 1931. Acta Psychiatr Scand 1991; 84: 142-149. 
14. Ayuso JL. Global burden of obsessive-compulsive disorder in the year 2000. Global Program on Evidence for Health Policy (GPE). Global Burden of Disease 2000. World Health Organization; 2000.

15. Berg CZ, Whitaker A, Davies M, Flament MF, Rapoport JL. The survey form of Leyton Obsessional Inventory child version: Norms from an epidemiological study. J Am Acad Child Adolesc Psychiatry 1989; 27(6): 759-763.

16. Marwa S. Prevalence of obsessive compulsive disorder among female secondary school students in Cairo [dissertation]. Ain Shams University; 2005.

17. El-Rakhawy M. Some variable related to the obsessional manifestations and symptoms in children attending psychiatric clinic. Leyton obsessional inventory child version [dissertation]. Ain Shams University; 1992.

18. Sheehan DV, Lecrubier Y, Sheehan KH, Amorim P, Janavs J, Weiller E, et al. The validity of Mini International Neuropsychiatric Interview (MINI). The development and validation of a structured diagnostic interview for DSM-IV and ICD-10. J Clin Psychiatry 1998; 59 (suppl20): 211-232.

19. Ibrahim M, Bishry Z, Hamed A. Comparison of Mini International Neuropsychiatric Interview for children (MINIKID) with the schedules for affective disorders and schizophrenia for school aged children, present and lifetime version (KSADS-PL): In Egyptian sample presenting with childhood disorders [dissertation]. Ain Shams University; 2002.

20. Ismail R. The prevalence of OCD in a sample of Egyptian students [dissertation]. Faculty of Medicine, ElAzhar University; 1998.

21. Hammoda M. Point prevalence rates of obsessive compulsive symptoms and obsessive-compulsive disorder in a sample of secondary school students in a rural area in Egypt. Current Psychiatry 2000; 7(2): 133-144.

22. Okasha A, Lotaief F, Ashour AM, el Mahalawy N, Seif el Dawla A, el-Kholy G. The prevalence of obsessive compulsive symptoms in a sample of Egyptian psychiatric patients. L'Encephale 2000; 26(4): 4-10.

23. Khalid S. Prevalence of psychological symptoms in Saudi secondary school girls in Abha, Saudi Arabia. Ann Saudi Med 2009; 29(4): 275-279.
24. Marwa S. Prevalence of obsessive compulsive disorder among female secondary school students in cairo [dissertation]. Ain Shams University; 2005.

25. Amin A. Prevalence of obsessive compulsive symptoms and disorder in a sample of Egyptian school children [dissertation]. Ain Shams University; 1998.

26. Okasha A. OCD in Egyptian adolescents: The effect of culture and religion. Psychiatric Times 2004; 21(5): 1-5.

27. El Nahas G. A follow up study of a sample of Egyptian OCD patients: Illness characteristics and outcome. Current Psychiatry 2007; 7(3): 368-380.

28. Rasim S, Ayse A. Sociodemographic and clinical characteristics of Turkish children and adolescents with obsessive compulsive disorder. Croat Med J 2002; 43: 324-329.

29. Murray B. Obsessive compulsive disorder in the community: An epidemiologic survey with clinical reappraisal. Am J Psychiatry 1997; 154: 1120-1126.

30. Pigot TA. Obssesive compulsive disorder: Symptoms overview and epidemiology. Bull Menninger Clin Fall 1998; 62(4): 24-32.

31. Khanna S, Srinath S. Childhood obsessive compulsive disorder. Psychopath 1989; 32: 47-54.

32. Zohar AH. An epidemiological study of obsessive compulsive disorder and related disorders in Israeli adolescents. $\mathrm{J}$ Am Acad Child Adolesc Psychiatry 1992; 31: 1057-1061.

33. Okasha A. The prevalence of obsessive compulsive symptoms in a sample of Egyptian psychiatric patients. L'Encephale 2000; 26(4): 4-10.

Corresponding author:

Ahmed Rady, MD

P.O. Box 518 - Alexandria 21511

Egypte

Tel.: +2 (0) 1282441053

E-mail: dr_ahmed_rady@yahoo.fr 